\title{
Mini-Mesohepatectomy for Colorectal Metastasis Invading the Middle Hepatic Vein at the Hepatocaval Confluence: Educational Video Demonstrating IOUS Use in Modern Hepatic Surgery
}

\author{
Masatoshi Makuuchi, $\mathbf{P h D}^{1}$ and Hideki Abe, $\mathbf{P h D}^{2}$ \\ ${ }^{1}$ Japanese Red Cross Medical Center, Tokyo, Japan; ${ }^{2}$ Ibaraki Prefectural Center Hospital, Kasama, Japan
}

Makuuchi's (MM's) first report on the use of intraoperative ultrasonography (IOUS) for hepatectomy was published in $1982 .^{1}$ Since then, MM has been stressing the importance of IOUS in the field of hepatic surgery, firmly believing that "those who conquer IOUS technique, conquer modern hepatic surgery." However, educational opportunities in IOUS for surgeons are limited, and depend greatly upon their individual and intentional effort. Torzilli et al., in their educational video manuscript, present us with a new surgical approach for tumors invading middle hepatic vein (MHV) at caval confluence, making full use of extensive and highly specialized ultrasonographical maneuvers. ${ }^{2}$ Ultrasound is here used to explore the liver for both staging and confirmation of tumor relations. The upto-date technique is also utilized in planning surgical strategy. $^{3-5}$

Expansion of surgical indication in diseases such as advanced hepatocellular carcinoma (HCC) and multiple bilobar colorectal cancer liver metastasis led surgeons to find technical solutions for resection of hepatic veins. Lack of portal blood perfusion at liver parenchyma induces liver dysfunction in the congested area, followed by atrophy. Kakazu et al. reported segment 6 congestion after resection of the right paramedian sector, and Lee et al. experienced necrosis of the right paramedian sector in living-donor liver transplantation (LDLT), leading to an idea of modified right liver graft. ${ }^{6-8}$ Experience with donor hepatectomies during LDLT showed congestion in the right paramedian of a right liver graft without the $\mathrm{MHV}$, also leading to graft

(C) Society of Surgical Oncology 2009

Published Online: 1 December 2009

M. Makuuchi, PhD

e-mail: makuuchi_masatoshi@med.jrc.or.jp dysfunction. ${ }^{7}$ Most focused their attention on making major resection safer, preparing patients preoperatively by adopting methods such as portal vein embolization, and promoting hepatic vein reconstruction. ${ }^{6,9}$ Liver-sparing surgery by means of hepatic vein reconstruction significantly decreased morbidity and mortality while still maintaining radicality in surgical resection. Although we are in no way denying the effectiveness of these approaches to the extent expected, by making full use of IOUS hepatic surgeons are given the option of achieving extreme parenchymal sparing in advanced diseases, normally considered suitable only for major resection or palliation, enhancing the feasibility of successful surgical approaches.

Use of preoperative computed tomography (CT) scan and three-dimensional reconstructions of the vessels reveals hepatic venous anastomosis. ${ }^{10}$ IOUS even reveals venous anastomosis at LDLT, using extended liver graft including the MHV, enabling MHV ligation at the junction of the left hepatic vein without regurgitation of the portal vein blood flow. ${ }^{11}$ Taking a step further, hepatic vein resection without its reconstruction based on intraoperative color Doppler findings, and more recently, on new, more sensitive ultrasound methods for blood flow disclosure, opens up a new perspective for liver surgery, providing important information elucidating the anatomy of the liver venous drainage such as the presence of communicating veins between adjacent hepatic veins. ${ }^{12}$ Indeed, the study of hepatic venous drainage was advanced dramatically with the introduction of ultrasound guidance.

With the use of e-flow ultrasound, Torzilli et al. evaluated the blood flow direction of the portal branches and hepatic veins, by applying finger compression to the hepatic vein. ${ }^{2}$ The amount of liver parenchyma to be spared was decided based upon the findings. 
Although it should be pointed out that their surgical approach awaits further scrutiny to validate its efficacy and safety, their technical proposal provides further evidence confirming that ultrasound guidance is progressively occupying a stronger role in the field of modern liver surgery. Educational video manuscripts such as theirs should be appreciated in an environment where educational opportunities are still few and lacking. ${ }^{2} \mathrm{We}$ are fortunate to have innovative surgeons such as those authors, understanding their responsibility in sharing technical knowledge and upto-date information where not all are privileged to be able to experience these up-to-date techniques at first hand.

\section{REFERENCES}

1. Makuuchi M, Hasegawa H, Yamazaki S. Intraoperative ultrasonic examination for Hepatectomy. Jpn J Clin Oncol. 1981;11:367-90.

2. Torzilli G, Botea F, Donadon M, Cimino M, Del Fabbro D, Palmisano A. Mini-mesohepatectomy for liver tumor invading the middle hepatic vein at the hepatocaval confluence (Video). Ann Surg Oncol. (in press).

3. Torzilli G, Del Fabbro D, Olivari N, Calliada F, Montorsi M, Makuuchi M. Contrast-enhanced intraoperative ultrasonography during liver surgery. Br J Surg. 2004;91:1165-7.

4. Torzilli G, Montorsi M, Del Fabbro D, Palmisano A, Donadon M, Makuuchi M. Ultrasonographically guided surgical approach to liver tumours involving the hepatic veins close to the caval confluence. Br J Surg. 2006;93:1238-46.

5. Torzilli G, Donadon M, Marconi M, Botea F, Palmisano A, Del Fabbro D, et al. Systematic extended right posterior sectionectomy: a safe and effective alternative to right hepatectomy. Ann Surg. 2008;247:603-11.

6. Kakazu T, Makuuchi M, Kawasaki S, Miyagawa S, Nakazawa Y, Kubota T, et al. Reconstruction of the middle hepatic vein tributary during right anterior segmentectomy. Surgery. 1995:117; 238-40.

7. Lee SG, Park KM, Hwang S, Lee YJ, Choi DN, Kim KH, et al. Congestion of the right liver graft in living donor liver transplantation. Transplantation. 2001;71:812-4.

8. Lee SG, Park KM, Hwang S, Kim KH, Choi DN, Joo SH, et al. Modified right liver graft from a living donor to prevent congestion. Transplantation. 2002;74:54-9.

9. Makuuchi M, Thai BL, Takayasu K, Takayama T, Kosuge T, Gunvén $\mathrm{P}$, et al. Preoperative portal embolization to increase safety of major hepatectomy for hilar bile duct carcinoma: a preliminary report. Surgery. 1990;107:521-7.

10. Satou S, Sugawara Y, Kokudo N, Makuuchi M. Preoperative detection of hepatic venous collaterals in right liver graft. Liver Transpl. 2005;11:708-9.

11. Cescon M, Sugawara Y, Sano K, Ohkubo T, Kaneko J, Makuuchi M. Right liver graft without middle hepatic vein reconstruction from a living donor. Transplantation. 2002;73:1164-6.

12. Sano K, Makuuchi M, Miki K, Maema A, Sugawara Y, Imamura $\mathrm{H}$, et al. Evaluation of hepatic venous congestion: proposed indication criteria for hepatic vein reconstruction. Ann Surg. $2002 ; 236: 241-7$ 